\title{
Dispersion Potentials between Atoms and Dielectric Bodies
}

\author{
F. Schuller \\ Laboratoire de Physique des Lasers, U.R.A. 282 du C.N.R.S., Université Paris-Nord, \\ 93430 Villetaneuse, France
}

Z. Naturforsch. 49a, 885-889 (1994); received July 6, 1994

\begin{abstract}
We derive a formula for the van der Waals interaction between an atom and a dielectric surface for both ground and excited state potentials. We compare our result with previously derived expressions involving the dielectric constant at purely imaginary frequencies. We also study the relationship between atom-dielectric and ordinary atom-atom van der Waals interaction.
\end{abstract}

Key words: Van der Waals potentials, Atoms, Dielectrics, Surface physics, Atomic spectroscopy.

\section{Introduction}

The theory of van der Waals interaction between an atom and a perfectly reflecting surface has been developed in the past by several authors for both dielectric and metallic surfaces [1-3]. However, in these early treatments it has always been assumed that the atom is in its ground state. Only recently has the case of excited state potentials been included in the theory [4]. This latter case is of importance in spectroscopic studies of selective reflection where frequency shifts due to v.d.W. interaction have been observed [5].

In this paper we present a simplified method for calculating v.d.W. potentials in both the ground and the excited state. Retardation effects [6] are not taken into account, mainly because these effects are not relevant for the experiments mentioned above.

We establish the equivalence between previously derived expressions involving the dielectric constant at purely imaginary frequencies and an expression where only real frequencies appear. Finally we present a test for the theory by linking it to the ordinary theory of binary v.d.W. potentials between atoms.

\section{The Image Theory}

According to electrostatics, the potential acting on an electric dipole located at a normal distance $z$ from a plane dielectric surface can be viewed as the interaction energy of the dipole with its electrical image in-

Reprint requests to Dr. F. Schuller, Laboratoire de Physique des Lasers, U.R.A. 282 du CNRS, Université Paris-Nord, Avenue Jean-Baptiste Clement, F-93430 Villetaneuse/France side the dielectric. In the case of a dipole behaving like a classical oscillator with

$$
\boldsymbol{\mu}(\omega)=\boldsymbol{\mu}_{0}(\omega) e^{-\mathrm{i} \omega t}
$$

the components of the image dipole $\boldsymbol{\mu}^{\prime}(\omega)$ are

$$
\begin{aligned}
& \mu_{x}^{\prime}(\omega)=-f(\omega) \mu_{x}(\omega), \\
& \mu_{y}^{\prime}(\omega)=-f(\omega) \mu_{y}(\omega), \\
& \mu_{z}^{\prime}(\omega)=f(\omega) \mu_{z}(\omega),
\end{aligned}
$$

where we have set

$$
f(\omega)=\frac{\varepsilon(\omega)-1}{\varepsilon(\omega)+1}
$$

with $\varepsilon(\omega)$ the frequency dependent dielectric constant.

The interaction energy of the dipole with the dielectric via the image dipole is then given by the expression

$$
V(z)=-\frac{1}{2(2 z)^{3}} f(\omega)\left(\mu_{x}^{2}+\mu_{y}^{2}+2 \mu_{z}^{2}\right),
$$

assuming that the frequency $\omega$ is sufficiently far from any resonance in the dielectric so that $f(\omega)$ can be regarded as real. By further assuming isotropy, so that on average $\mu_{x}^{2}=\mu_{y}^{2}=\mu_{z}^{2}=\frac{1}{3}|\boldsymbol{\mu}|^{2}$, we thus have

$$
V(z)=-\frac{1}{12 z^{3}} f(\omega)|\boldsymbol{\mu}|^{2} .
$$

Note that in the case where the dielectric is replaced by a metallic surface we have $f(\omega)=1$ and hence

$$
V(z)=-\frac{|\mu|^{2}}{12 z^{3}}
$$

a well-known result [7]. In the case of an atom one must consider fluctuations of the atomic dipole due to 
its interaction with the vacuum field. Then the time dependent dipole $\boldsymbol{\mu}(t)$ can be expressed in terms of Fourier components with Fourier amplitudes given by

$$
\hat{\mu}_{\alpha}(\omega)=\frac{1}{2 \pi} \int_{-\infty}^{+\infty} \mu_{\alpha}(t) e^{i \omega t} \mathrm{~d} t
$$

with $\alpha=x, y, z$ designating cartesian components of the vector $\boldsymbol{\mu}$. By applying the same procedure to the image dipole we write

$$
\mu_{\alpha}^{\prime}(t)=\int_{-\infty}^{+\infty} \hat{\mu}_{\alpha}^{\prime}(t) e^{-i \omega t} \mathrm{~d} \omega
$$

We now assume that Fourier components of the dipole and its image are related according to (2), i.e. we write

with

$$
\hat{\mu}_{\alpha}^{\prime}(\omega)=p_{\alpha} f(\omega) \hat{\mu}_{\alpha}(\omega)
$$

$$
p_{x}=p_{y}=-1, \quad p_{x}=1 .
$$

By combining (7), (8) and (9) we then have

$\mu_{\alpha}^{\prime}(t)=p_{\alpha} \frac{1}{2 \pi} \int_{-\infty}^{+\infty} f(\omega) \mathrm{d} \omega \int_{-\infty}^{+\infty} \mu_{\alpha}\left(t^{\prime}\right) e^{-i \omega\left(t-t^{\prime}\right)} \mathrm{d} t^{\prime}$.

Now, because of the special properties of the dielectric function $\varepsilon(\omega)$ contained in $f(\omega)$, the integral in (10) is only non zero for values $t-t^{\prime}>0$ (this fact can also be viewed as the expression of the causality principle, meaning that the image dipole at time $t$ can only depend on values $t^{\prime}$ anterior to $t$ ). By introducing the new integration variable $t-t^{\prime}=\tau$, we can write (10) in the form

$\mu_{\alpha}^{\prime}(t)=p_{\alpha} \frac{1}{2 \pi} \int_{-\infty}^{+\infty} f(\omega) \mathrm{d} \omega \int_{0}^{+\infty} \mu_{\alpha}(t-\tau) e^{-i \omega \tau} \mathrm{d} \tau$.

Considering the interaction with the dielectric, we start from the expression

$$
V=-\frac{1}{2} \frac{1}{(2 z)^{3}} \sum_{\alpha} q_{\alpha}\left\langle\mu_{\alpha}(t) \mu_{\alpha}^{\prime}(t)\right\rangle,
$$

where the brackets designate a time average of the expectation value of the operator product for the atomic state to which the potential refers. The quantities $q_{\alpha}$, with $q_{x}=q_{y}=-1, q_{z}=2$, are the usual coefficients arising for dipole-dipole interaction. If we substitute now for $\mu_{\alpha}^{\prime}(t)$ the expression (11), we arrive at the following result for the interaction energy: with

$$
w_{\alpha}=q_{\alpha} p_{\alpha} ; \quad w_{x}=w_{y}=1, w_{z}=2 .
$$

The second integral in this expression can be interpreted as the Laplace-Fourier transform of the autocorrelation function of the fluctuating dipole moment of the atom. This integral is therefore related to the profiles associated with transitions from some initial state $|i\rangle$ to all possible intermediate states $|f\rangle$ connected to $|i\rangle$ by non zero dipole matrix elements $\boldsymbol{\mu}_{i f}=\langle i|\boldsymbol{\mu}| f\rangle$. We therefore write

$\int_{0}^{+\infty}\left\langle\mu_{\alpha}(t) \mu_{\alpha}(t-\tau)\right\rangle e^{i \omega \tau} \mathrm{d} \tau=\pi \sum_{f}\left|\mu_{\alpha}^{i f}\right|^{2} \Phi_{i f}(\omega)$,

where the function

$$
\Phi_{i f}(\omega)=\Phi_{i f}^{-}\left(\omega_{f i}-\omega\right)+\Phi_{i f}^{+}\left(\omega_{f i}+\omega\right)
$$

involves the following lorentzian profiles:

$$
\Phi_{i f}^{-,+}(\Omega)=\left(\frac{\gamma_{i f}}{\Omega^{2}+\gamma_{i f}^{2}}+i \frac{\Omega}{\Omega^{2}+\gamma_{\text {if }}^{2}}\right) / \pi
$$

with

$\Omega=\omega_{f i}-\omega$ for $\Phi^{-}$and $\Omega=\omega_{f i}+\omega$ for $\Phi^{+}$.

Here $\omega_{f i}$ is the frequency and $\gamma_{i f}$ the natural line width of the considered transition. Defined in this way, $\Phi_{i f}$ has the property

$$
\Phi_{i f}(-\omega)=\Phi_{i f}^{*}(\omega)
$$

With the definitions (14) and (15) the interaction energy takes the form

$V=-\frac{1}{2} \frac{1}{(2 z)^{3}} \sum_{\alpha f} w_{\alpha}\left|\mu_{\alpha}^{i f}\right|^{2} \frac{1}{2} \int_{-\infty}^{+\infty} f(\omega) \Phi_{i f}(-\omega) \mathrm{d} \omega$.

By restricting the range of integration to the positive frequency domain the integral in this expression can be written as

$$
\begin{aligned}
I & =\int_{-\infty}^{+\infty} f(\omega) \Phi_{i f}(-\omega) \mathrm{d} \omega \\
& =\int_{0}^{+\infty} f(\omega) \Phi_{i f}(-\omega) \mathrm{d} \omega+\int_{0}^{+\infty} f(-\omega) \Phi_{i f}(\omega) \mathrm{d} \omega .
\end{aligned}
$$

$$
V=-\frac{1}{2} \frac{1}{(2 z)^{3}} \sum_{\alpha} w_{\alpha} \frac{1}{2 \pi} \int_{-\infty}^{+\infty} f(\omega) \mathrm{d} \omega \int_{0}^{+\infty}\left\langle\mu_{\alpha}(t) \mu_{\alpha}(t-\tau)\right\rangle e^{-i \omega \tau} \mathrm{d} \tau
$$


By noticing further that $\varepsilon(-\omega)=\varepsilon^{*}(\omega)$ and hence $f(-\omega)=f^{*}(\omega)$, this integral can also be written as

$$
\begin{aligned}
I= & 2 \int_{0}^{+\infty} \operatorname{Re} f(\omega) \operatorname{Re} \Phi_{i f}(\omega) \mathrm{d} \omega \\
& +2 \int_{0}^{+\infty} \operatorname{Im} f(\omega) \operatorname{Im} \Phi_{i f}(\omega) \mathrm{d} \omega,
\end{aligned}
$$

where the property (17) has been used.

In previous derivations of the van der Waals interaction energy the width parameter $\gamma$ has always been considered as an infinitesimal quantity. In this case we can make use of the relations

$$
\frac{\gamma}{\Omega^{2}+\gamma^{2}} \rightarrow \pi \delta(\Omega) ; \frac{\Omega}{\Omega^{2}+\gamma^{2}} \rightarrow P P \frac{1}{\Omega},
$$

involving a delta function and a principal value.

In this limiting case our function $\Phi_{i f}(\omega)$ takes the form

$\Phi_{i f}(\omega)=\delta\left(\omega_{f i}-\omega\right)+\delta\left(\omega_{f i}+\omega\right)+\frac{i}{\pi} P P \frac{2 \omega_{f i}}{\omega_{f i}^{2}-\omega^{2}}$.

By substituting this result into (18), we obtain for the interaction energy the following final expression:

$$
\begin{aligned}
V= & -\frac{1}{2} \frac{1}{(2 z)^{3}} \sum_{\alpha f}\left|\mu_{\alpha}^{i f}\right|^{2} w_{\alpha} \\
& \cdot\left\{\frac{2}{\pi} P P \int_{0}^{+\infty} \operatorname{Im} f(\omega) \frac{\omega_{f i}}{\omega_{f i}^{2}-\omega^{2}} \mathrm{~d} \omega+\operatorname{Re} f\left(\left|\omega_{f i}\right|\right)\right\} .
\end{aligned}
$$

Note that if anisotropy is neglected, this expression becomes

$$
V=-\frac{1}{12 z^{3}} \sum_{f}\left|\mu_{i f}\right|^{2}\{\ldots\} .
$$

In the next paragraph we show that in the case where $|i\rangle$ is the ground state, so that $\omega_{f i}$ is always positive, this expression is equivalent with the one derived by Mavroyannis [1]. In the case of an excited state potential, where some of these frequencies are negative, our expression reproduces the results of [4].

\section{Imaginary Frequencies}

The expression for the interaction potential in terms of imaginary frequencies derived by Mavroyannis [1] is based on the assumption that the atom interacting with the dielectric surface is in its ground state. A similar expression, but which is also valid for atoms in

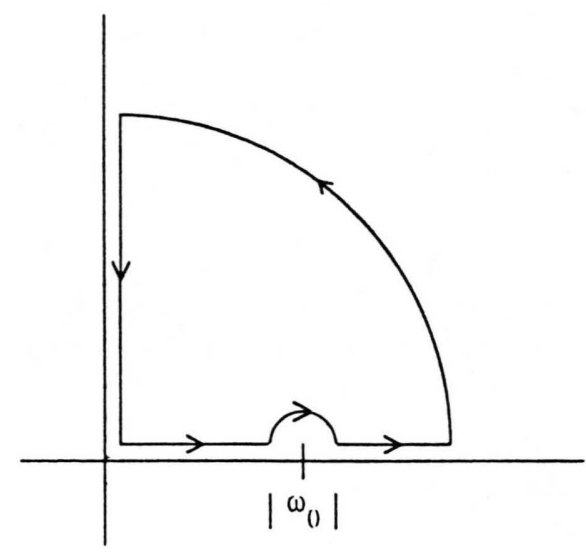

Fig. 1. Integration path for the integral in (17).

an excited state, can be derived from our general formula (23) by performing an integration in the complex plane.

Consider an integral of the type

$$
J=\oint f(z) \frac{\omega_{0}}{\omega_{0}^{2}-z^{2}} \mathrm{~d} z
$$

over the closed contour represented on Figure 1. This integral can be written in the form

$J=P \int_{0}^{\infty} f(\omega) \frac{\omega_{0}}{\omega_{0}^{2}-\omega^{2}} \mathrm{~d} \omega-\int_{0}^{\infty} f(i \mathrm{u}) \frac{\omega_{0}}{\omega_{0}^{2}+u^{2}} i \mathrm{~d} u+\Gamma$,

where $\Gamma$ represents the contribution from the small half circle around the singularity $z=\left|\omega_{0}\right|$. According to whether $\omega_{0}$ is positive or negative we find for $\Gamma$ the values

$$
\begin{array}{ll}
\Gamma=\frac{i \pi}{2} f\left(\omega_{0}\right), & \omega_{0}>0, \\
\Gamma=-\frac{i \pi}{2} f\left(-\omega_{0}\right), & \omega_{0}<0 .
\end{array}
$$

In the first case (26) then yields the expression

$$
\begin{aligned}
P & \int_{0}^{\infty} \operatorname{Im} f(\omega) \frac{\omega_{0}}{\omega_{0}^{2}-\omega^{2}} \mathrm{~d} \omega+\frac{\pi}{2} \operatorname{Re} f\left(\omega_{0}\right) \\
& =\int_{0}^{\infty} f(i u) \frac{\omega_{0}}{\omega_{0}^{2}+u^{2}} \mathrm{~d} u
\end{aligned}
$$

if only real parts are considered. Note that, as can be shown, the integral on the r.h.s. of this equation is always real. 
With this identity, (23) yields for the interaction potential the expression

$V(z)=-\frac{1}{6 \pi z^{3}} \sum_{f}\left|\boldsymbol{\mu}_{i f}\right|^{2} \int_{0}^{\infty} \frac{\varepsilon(i u)-1}{\varepsilon(i u)+1} \frac{\omega_{f i}}{\omega_{f i}^{2}+u^{2}} \mathrm{~d} u$,

which is the result of [1].

In the second case, i.e. for $\omega_{0}<0$, the change in the value of $\Gamma$ can be accounted for if we add on the r.h.s. of (28) twice the value $\frac{\pi}{2} \operatorname{Re} f\left(\omega_{0}\right)$. We thus have

$$
\begin{array}{r}
P \int_{0}^{\infty} \operatorname{Im} f(\omega) \frac{\omega_{0}}{\omega_{0}^{2}-\omega^{2}} \mathrm{~d} \omega+\frac{\pi}{2} \operatorname{Re} f\left(\omega_{0}\right) \\
\quad=\int_{0}^{\infty} f(i u) \frac{\omega_{0}}{\omega_{0}^{2}+u^{2}} \mathrm{~d} u+\pi \operatorname{Re} f\left(\omega_{0}\right) .
\end{array}
$$

From this result we see that, in the case where the initial state $i$ is an excited one so that transitions to lower states can occur, we must generalize (29) by writing it as

$$
\begin{aligned}
V(z)=-\frac{1}{6 \pi z^{3}} \sum_{f}\left|\boldsymbol{\mu}_{i f}\right|^{2} & \left\{\int_{0}^{\infty} \frac{\varepsilon(i u)-1}{\varepsilon(i u)+1} \frac{\omega_{f i}}{\omega_{f i}^{2}+u^{2}} \mathrm{~d} u\right. \\
+ & \left.\Theta\left(-\omega_{f i}\right) \pi \operatorname{Re} \frac{\varepsilon\left(\left|\omega_{f i}\right|\right)-1}{\varepsilon\left(\left|\omega_{f i}\right|\right)+1}\right\} .
\end{aligned}
$$

An identical result has been derived previously in [4].

The two equivalent equations (31) and (23) cover the general situation of both ground and excited state potentials, whereas (29) only applies if all the transition frequencies $\omega_{f i}$ are positive.

\section{The Binary Interaction Limit}

As an illustrative example let us consider a simplified, somewhat academic model where we assume that the atom-dielectric potential is just the sum over binary interactions of the type

$$
V_{6}(R)=-\frac{C_{6}}{R^{6}}
$$

between the atom and all the individual atoms of the dielectric. This means that atomic interactions within the dielectric are neglected so as if the latter were a dilute gas. By performing an integration over all the distances between the atom and the homogeneously distributed atoms of the dielectric, one obtains for the potential as function of the normal distance $z$ the result

$$
V(z)=-\frac{C_{z}}{z^{3}}
$$

with the following relation between $C_{6}$ and $C_{z}$ :

$$
C_{6}=\frac{6}{\pi} \frac{1}{N} C_{z},
$$

where $N$ is the number density of atoms in the dielectric.

Making the binary interaction approximation means that one can write

$$
\frac{\varepsilon-1}{\varepsilon+1} \rightarrow \frac{1}{2}(\varepsilon-1)=2 \pi N \alpha_{D},
$$

where $\alpha_{D}$ is the polarizability of one of the atoms of the dielectric. By introducing the usual expression in terms of oscillator strength

$$
\alpha_{D}=\frac{e^{2}}{m} \sum_{d^{\prime}} \frac{f_{d d^{\prime}}}{\omega_{d^{\prime} d}^{2}-\omega^{2}},
$$

where $d$ labels the ground state and $d^{\prime}$ the final state of the virtual transitions with oscillator strength $f_{d d^{\prime}}$, we obtain with the approximation (35) the relation

$$
\frac{\varepsilon(i u)-1}{\varepsilon(i u)+1}=2 \pi N \frac{e^{2}}{m} \sum_{d^{\prime}} \frac{f_{d d^{\prime}}}{\omega_{d^{\prime} d}^{2}+u^{2}} .
$$

An elementary integration then yields for the integral appearing in (31)

$$
\begin{aligned}
& \int_{0}^{\infty} \frac{\varepsilon(i u)-1}{\varepsilon(i u)+1} \frac{\omega_{f i}}{\omega_{f i}^{2}+u^{2}} \mathrm{~d} u \\
& =\pi^{2} N \frac{e^{2}}{m} \sum_{d^{\prime}} f_{d d^{\prime}} \frac{\omega_{f i}}{\omega_{d^{\prime} d}^{2}-\omega_{f i}^{2}}\left(-\frac{1}{\omega_{d^{\prime} d}}+\frac{1}{\left|\omega_{f i}\right|}\right) .
\end{aligned}
$$

From (35) and (36) we also have

$$
\pi \operatorname{Re} \frac{\varepsilon\left(\omega_{f i}\right)-1}{\varepsilon\left(\omega_{f i}\right)+1}=2 \pi^{2} N \frac{e^{2}}{m} \sum_{d^{\prime}} f_{d d^{\prime}} \frac{1}{\omega_{d^{\prime} d}^{2}-\omega_{f i}^{2}} .
$$

Hence the bracket in (31) can be written in the form

$$
\begin{aligned}
\{\}= & 2 \pi^{2} N \frac{e^{2}}{m} \sum_{d^{\prime}} f_{d d^{\prime}} \frac{1}{\omega_{d^{\prime} d}^{2}-\omega_{f i}^{2}} \\
& \cdot\left[\frac{1}{2} \omega_{f i}\left(-\frac{1}{\omega_{d^{\prime} d}}+\frac{1}{\left|\omega_{f i}\right|}\right)+\Theta\left(-\omega_{f i}\right)\right] .
\end{aligned}
$$

Then a simple calculation shows that in both cases, i.e. $\omega_{f i}>0$ and $\omega_{f i}<0$, we have for \{\} in (40) the same 
expression, namely

$$
\{\}=\pi^{2} N \frac{e^{2}}{m} \sum_{d^{\prime}} \frac{f_{d d^{\prime}}}{\omega_{d^{\prime} d}} \frac{1}{\omega_{d^{\prime} d}+\omega_{f i}} .
$$

Hence we obtain for the potential

$$
V(z)=-\frac{\pi N}{6 z^{3}} \frac{e^{2}}{m} \sum_{f d^{\prime}}\left|\boldsymbol{\mu}_{i f}\right|^{2} \frac{f_{d d^{\prime}}}{\omega_{d^{\prime} d}} \frac{1}{\omega_{d^{\prime} d}+\omega_{f i}} .
$$

By introducing for the outside atom the oscillator strength defined by the relation

$$
f_{i f}=\frac{2}{3} \frac{m}{\hbar e^{2}} \omega_{f i}\left|\boldsymbol{\mu}_{i f}\right|^{2},
$$

we write $V(z)$ in the form

$$
V(z)=-\frac{\pi}{4} \hbar \frac{e^{4}}{m^{2}} N \frac{1}{z^{3}} \sum_{f d^{\prime}} \frac{f_{i f} f_{d d^{\prime}}}{\omega_{f i} \omega_{d^{\prime} d}\left(\omega_{f i}+\omega_{d^{\prime} d}\right)}
$$

This result, combined with (34), yields for the van der Waals constant $C_{6}$ for binary interactions between atoms of different species the old London formula quoted in classical references [8, 9].

[1] C. Mavroyannis, Mol. Phys. 6, 593 (1963).

[2] A. D. Mc Lachlan, Proc. Roy. Soc. London A 271, 387 (1963).

[3] A. D. Mc Lachlan, Mol. Phys. 6, 423 (1963); 7, 381 (1964).

[4] J. M. Wylie and J. E. Sipe, Phys. Rev. A 30, 1185 (1984); Phys. Rev. A 32, 2030 (1985).

[5] M. Chevrollier, M. Fichet, M. Oria, G. Rahmat, D. Bloch, and M. Ducloy, J. Phys. II Fr. 2, 631 (1992).

\section{Conclusion}

We have derived a formula for the long range interaction potential between an atom and a plane dielectric surface in terms of the dielectric constant $\varepsilon$ as function of the real frequency $\omega$. In this way the potential can be calculated for realistic situations, for instance if only tabulated values of $\varepsilon(\omega)$ are available. The formula applies to both ground and excited state potentials. Moreover we have established the equivalence between this formula and a recent result based on the method of Mavroyannis where the frequency domain is shifted to the imaginary axis.

Finally, we have demonstrated the complete identity between the results obtained by this latter method and those of the old quantum theory of interatomic potentials in the limit of an infinitely dilute medium.

\section{Acknowledgements}

The author wants to thank G. Nienhuis and M. Ducloy for very helpful discussions.

[6] H. B. G. Casimir and D. Polder, Phys. Rev. 73, 360 (1948)

[7] J. E. Lennard-Jones, Trans. Faraday Soc. 28, 334 (1932).

[8] J. O. Hirschfelder, C. F. Curtiss, and R. B. Bird, Molecular Theory of Gases and Liquids, John Wiley, New York 1974.

[9] E. Lindholm, Thesis, Uppsala (1942). 